\title{
Robust Vessel Tree Modeling
}

\author{
M. Akif Gülsün and Hüseyin Tek \\ Imaging and Visualization, Siemens Corporate Research, Princeton, NJ, USA \\ akif.gulsun@siemens.com, Huseyin.Tek@siemens.com
}

\begin{abstract}
In this paper, we present a novel method for extracting center axis representations (centerlines) of blood vessels in contrast enhanced (CE)-CTA/MRA, robustly and accurately. This graph-based optimization algorithm which employs multi-scale medialness filters extracts vessel centerlines by computing the minimum-cost paths. Specifically, first, new medialness filters are designed from the assumption of circular/elliptic vessel cross-sections. These filters produce contrast and scale independent responses even the presence of nearby structures. Second, they are incorporated to the minimum-cost path detection algorithm in a novel way for the computational efficiency and accuracy. Third, the full vessel centerline tree is constructed from this optimization technique by assigning a saliency measure for each centerline from their length and radius information. The proposed method is computationally efficient and produces results that are comparable in quality to the ones created by experts. It has been tested on more than 100 coronary artery data set where the full coronary artery trees are extracted in 21 seconds in average on a $3.2 \mathrm{GHz} \mathrm{PC}$.
\end{abstract}

\section{Introduction}

Modeling of vascular structures from contrast enhanced (CE) Computer Tomography Angiography(CTA) and Magnetic Resonance Angiography (MRA) is often a necessary task for diagnosis, treatment planning and follow-up studies in clinical applications. While recent technological advances in image acquisition devices e.g., new multi-detector CT machines, increase the spatial resolution of image data significantly, accurate and timely modeling of blood vessels is a still challenging task in many applications. Specifically, $(i)$ intensity contrast may change drastically along a single vessel; $(i i)$ vessels may touch nearby bright structures such as bone or other vessels; (iii) a single vessel tree can have large and small vessels i.e., significant scale change; $(i v)$ local vessel structure may deviate from a tubular structure due to the presence of pathologies such as stenosis. In clinical applications, a vessel modeling algorithm must be able to handle these imaging issues and still produce robust and accurate results in short time e.g. in few seconds for a single vessel, which are the main goals of our work. Some of the previous work on vessel centerline modeling methods include vesselness-based methods 651211] and medialness filters based methods 21118].

In this paper, we propose a new framework for the extraction of center-axis representation of vessels from CTA, MRA and 3D-X ray. Specifically, first, a 
novel medialness measure based on 2D multi-scale cross-sectional models is introduced. This new measure is contrast and scale independent and it works well in the presence of nearby bright structures such as bones or other vessels. Second, we present a minimal path detection method working on a discrete grid where the cost of graph edges are computed from multi-scale medialness filters. Third, the full vessel centerline tree from a single seed is extracted by a post-processing algorithm which uses the length and scale of vessel centerlines. In general, the proposed method can produce centerline model(s) for a vessel segment as well as the full vessel tree. In addition, it is capable of capturing different size of vessel branches, crossing over stenosis. Moreover, it is computationally efficient, i.e., it takes less than 21 seconds in average on a typical PC to obtain the centerlines of the full coronary artery trees including smaller branches. Furthermore, it works well for different types of vessels such as carotid, peripheral, aorta obtained from different imaging modalities such as CTA, MRA and 3D-X ray. We have tested it on more than 100 coronary CTA data as well as on several other types of vessels and the computed results were comparable to the expert created ones.

\section{Medialness Measure from 2D Cross-Sectional Models}

In this paper, our goal is to obtain the centerline representations of vessels directly from images without creating a binary vessel mask. Specifically, we propose a novel technique for computing medialness measure which is based on multiscale cross-sectional vessel modeling. Blood vessels in CTA/MRA have typically circular/elliptic shapes in cross-sectional views even though local variations on them are not too uncommon due to the presence of nearby vessels or pathologies. Ideally, 2D cross-sectional vessel profile consists of a circular/elliptic bright disk and darker ring around it. Our medialness measure uses this circularity assumption and edge responses obtained from multi-scale filters. Specifically, our medialness response, $m\left(\boldsymbol{x}_{\mathbf{0}}\right)$ at $\boldsymbol{x}_{\mathbf{0}}$, is computed from a circle $C\left(\boldsymbol{x}_{\mathbf{0}}, R\right)$ centered at $\boldsymbol{x}_{\mathbf{0}}$, with radius $R$, and is given by

$$
m\left(\boldsymbol{x}_{\mathbf{0}}\right)=\max _{R}\left\{\frac{1}{N} \sum_{i=0}^{N-1} E\left(\boldsymbol{x}_{\mathbf{0}}+R \boldsymbol{u}(\mathbf{2} \pi \boldsymbol{i} / \boldsymbol{N})\right)\right\}
$$

where $\boldsymbol{u}(\alpha)=\sin (\alpha) \boldsymbol{u}_{\mathbf{1}}+\cos (\alpha) \boldsymbol{u}_{\mathbf{2}}$ and $\boldsymbol{u}_{\boldsymbol{1}}$ and $\boldsymbol{u}_{\boldsymbol{1}}$ defines a $2 \mathrm{D}$ plane. $E$ measures the normalized edge response which is described below. Krissian et. al., 7] proposed a similar medialness measure where the cross-sectional plane is computed from the eigenvectors of Hessian matrix.

Let us consider a 1-D intensity profile $I(x)$ along a ray $\boldsymbol{u}_{\boldsymbol{\alpha}}$ on a cross-sectional plane of a vessel starting from the location $\boldsymbol{x}_{\mathbf{0}}$. Suppose that $\boldsymbol{x}_{\mathbf{0}}$ is the center of the vessel with a radius $R$. Then the cross-sectional boundary of the vessel along the ray should occur at $\left(\boldsymbol{x}_{\mathbf{0}}+R \boldsymbol{u}_{\boldsymbol{\alpha}}\right)$ where the gradient of $I(x)$ has a maxima and the second derivative of $I(x)$ has a zero-crossing. We propose to use the gradient, $\nabla_{\sigma} I(x)$ for measuring responses at vessel boundaries, in which $\sigma$ corresponds to the spatial scale of the vessel boundary. These gradients are normalized based on their filter sizes, $\sigma$ to obtain comparable results between different scales. In general, filter sizes are often selected from the size of vessels 
for computing gradient responses [7, i.e., larger spatial filters for large vessels. It should be noted that vessel scale, namely $R$ and boundary scale, $\sigma$ are not always related. For example, the boundary of a large vessel can be detected better with small size filters when such vessels are surrounded by other bright structures. Similarly, it is possible that small scale vessels can have long diffused boundaries which cannot be accurately detected via small scale filters.

Let us now define the boundary measure along a ray $\boldsymbol{u}_{\boldsymbol{\alpha}}$ at the location $x$,

$$
b(x)=\max _{\sigma}\left\{\left(\left|\nabla_{\sigma} I(x)\right|\right)\right\} \operatorname{sign}\left(\nabla_{\sigma} I(x)\right)
$$

where $\operatorname{sign}(x)$ is used to distinguish the rising (dark to bright changes) and falling edges (bright to dark changes). Observe that this boundary measure, $\nabla_{\sigma} I$ is contrast dependent, i.e., it obtains higher values from high contrast vessels and lower values from low contrast vessels, respectively. Unfortunately, vessels may have significant intensity variations on them - especially vessels in MRA and small size vessels in CTA. In addition, boundaries of bones, calcifications in CTA and vessels next to airways can have strong gradients which usually effect the response of medialness filters. We, in fact, believe that medialness responses should be contrast independent, which can be accomplished by normalizing the boundary measure via the highest gradient obtained for different $R$ values along the ray. Mathematically, we define a normalized boundary measure as $\hat{b}(x)=$ $b(x) / b_{\max }$ where $b_{\max }$ is the maximum falling edge response along $I(x)$ for $x=$ $\left\{\boldsymbol{x}_{\mathbf{0}}+R_{\min } \boldsymbol{u}_{\boldsymbol{\alpha}}, . ., \boldsymbol{x}_{\mathbf{0}}+R_{\max } \boldsymbol{u}_{\boldsymbol{\alpha}}\right\}$ and $R_{\min }$ and $R_{\max }$ are the minimum and maximum vessel scales, respectively.

Since the size of vessels to be modeled is not known a priori, our method searches for strong edge responses at the different locations along the ray $u_{\alpha}$ with different $R, R \in\left[R_{\min }, R_{\max }\right]$. However, observe that for large values of $R$ this produces strong boundary responses at locations which are outside the vessel. In general, there should not be any strong rising edge between $\boldsymbol{x}_{\mathbf{0}}$ and $\boldsymbol{x}_{\mathbf{0}}+R \boldsymbol{u}_{\boldsymbol{\alpha}}$ where the boundary is searched. If there exists such a strong rising edge, it probably means that the point $\boldsymbol{x}_{\mathbf{0}}$ is outside the vessel, thus it should have a lower medialness measure. This is accomplished by first computing the maximum rising boundary response up to the location $\boldsymbol{x}_{\mathbf{0}}+R \boldsymbol{u}_{\boldsymbol{\alpha}}$ along the ray and then subtracting this value from the response obtained at $\boldsymbol{x}_{\mathbf{0}}+R \boldsymbol{u}_{\boldsymbol{\alpha}}$. Based on these modifications, the final edge response along a ray, $\boldsymbol{u}_{\boldsymbol{\alpha}}$, starting from at $\boldsymbol{x}_{\mathbf{0}}, E\left(\boldsymbol{x}_{\mathbf{0}}+R \boldsymbol{u}_{\boldsymbol{\alpha}}\right)$ is given as

$$
E\left(\boldsymbol{x}_{\mathbf{0}}+R \boldsymbol{u}_{\boldsymbol{\alpha}}\right)=\frac{\max \left(-b\left(\boldsymbol{x}_{\mathbf{0}}+R \boldsymbol{u}_{\boldsymbol{\alpha}}\right)-\min _{x \in\left\{\boldsymbol{x}_{\mathbf{0}}, \boldsymbol{x}_{\mathbf{0}}+R \boldsymbol{u}_{\boldsymbol{\alpha}}\right\}}(b(x), 0), 0\right)}{\max _{x \in\left(\boldsymbol{x}_{\mathbf{0}}+R_{\min } \boldsymbol{u}_{\boldsymbol{\alpha}}, \boldsymbol{x}_{\mathbf{0}}+R_{\max } \boldsymbol{u}_{\boldsymbol{\alpha}}\right)}(-b(x), 1)}
$$

Observe that our medialness measure integrates edge responses along different size circles, thus, it is not sensitive to the isolated noise on a particular location. In general, this proposed technique has two major contributions: First, its response characteristics are very close to the ones that may be expected from an ideal medialness filter. Specifically, the proposed medialness measure gives strong responses at the center of a vessel and responses drop rapidly towards vessel boundaries and very small responses are obtained in non-vascular areas, Figure 1. Also, the presence of bright structures does not have strong impact on 

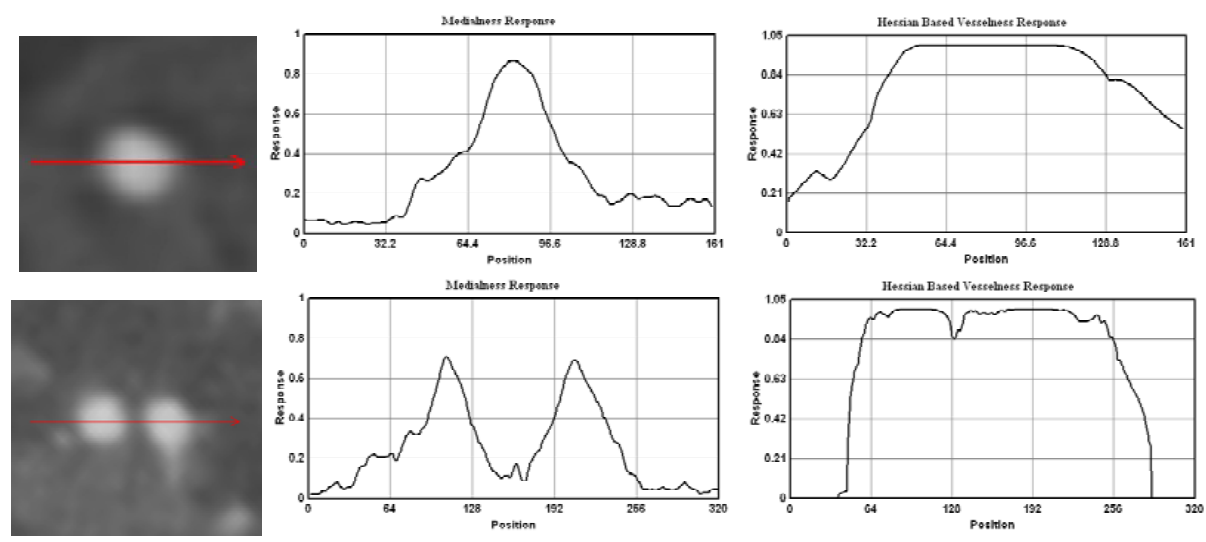

Fig. 1. This figure illustrates the medialness responses along a ray on two different examples obtained from our method (middle column) and the Hessian-based method (right column). Observe that unlike Hessian based methods, our technique gives low responses between two nearby vessels.

(a)

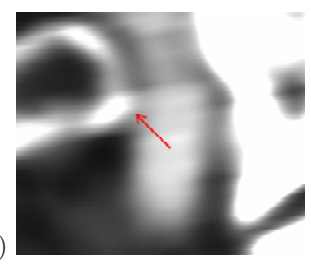

(b)

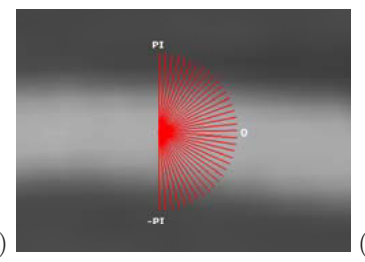

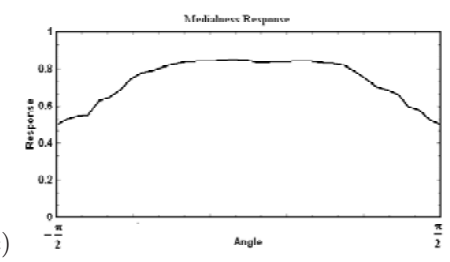

Fig. 2. (a) This figure illustrates the vessel direction obtained from the Hessian-based method. Observe that the presence of bone erroneously effects the direction of the vessel. (b) and (c) Medialness measure of a point when our medialness filters are applied on different orientations.

the responses. In fact, unlike Hessian based techniques [6 512 11], our approach gives low response between two nearby vessels, Figure 11 Second, our technique does not require the estimation of the vessel direction. Other techniques often uses the eigenvectors of the Hessian matrix to determine the vessel direction. However, bright structures such as bone, vessels, calcification, which are close to the vessel of interest can erroneously effect the direction of the vessel, thus medialness measure, as well, Figure 2 2 . Our proposed filtering technique produces higher responses when they are computed from orthogonal planes and lower responses when they are computed from oblique planes, Figure $2 \mathrm{~b}, \mathrm{c}$.

\section{Local Center-Axis from Graph-Based Optimization}

Medialness map of an image alone cannot be used in analyzing vessels without additional post-processing. Instead, they are constructed to obtain vessel 
center axis representations which are very useful in visualizing vessels in curved (or ribbon - flattened) multi-planar reformatting (MPR), in quantification of pathologies, in navigation during endovascular interventional treatments, etc. Local vessel center axis between two user selected points is often sufficient for analyzing a segment of a vessel quickly in clinical applications. Thus, in this section, we propose a method for extracting such local center axis representations by integrating the medialness map in a discrete optimization framework. Specifically, we seek to obtain a curve $C(s)$ (center axis) between points $p_{0}$ and $p_{1}$ which travels through the center of a vessel. This problem can be successfully solved by the minimum-cost path detection algorithms [3810]: Let $E(C)$ be the total energy along a curve $C$

$$
E(C)=\int_{\Omega}(P(C(s))+w) d s
$$

where $P(C)$ is called potential, $w$ is the regularization term and $s$ is the arch length, i.e., $\|C(s)\|^{2}=1$. In vessel centerline extraction methods, potential $P(x)$ at $x$ corresponds to the inverse of a medialness measure at that location, namely, $P(x)=\frac{1}{m(x)}$. Let $A_{p_{0}, p_{1}}$ represents the set of all curves between $p_{0}$ and $p_{1}$. The curve with total minimum energy can be computed from the minimumaccumulative cost, $\phi(p)$ which measures the minimal energy at $p$ integrated along a curve starting from the point $p_{0}$ :

$$
\phi(p)=\inf _{A_{p_{0}, p_{1}}}\{E(C)\}
$$

This type of minimization problems has been studied extensively in computer vision for different problems, e.g., segmentation. They are usually solved by either Dijkstra's algorithm [4] or Fast Marching methods 9]. In this paper, we propose to use Dijkstra's algorithm for solving equation (5) in a discrete domain. Specifically, let $G=(N, E)$ be a discrete graph where $N$ and $E$ represent nodes and edges, respectively. The minimum-accumulative cost at the node $P_{i j}$ for a four connected 2D graph is then given by

$$
\phi\left(P_{i j}\right)=\min \left(\phi\left(P_{i-1 j}\right)+C_{(i-1) j}^{i j}, \phi\left(P_{i+1 j}\right)+C_{(i+1) j}^{i j}, \phi\left(P_{i j-1}\right)+C_{i(j-1)}^{i j}, \phi\left(P_{i j+1}\right)+C_{i(j+1)}^{i j}\right)
$$

where, for example, $C_{(i-1) j}^{i j}$ corresponds to the cost of propagation from point $P_{(i-1) j}$ to $P_{i j}$ which is obtained from the inverse of medialness measure. This above algorithm can be easily implemented by first setting minimumaccumulative cost of all nodes to infinity (or a large value) and then using an explicit discrete front propagation method where propagation always takes places from the minimum value to its neighboring nodes. In our implementation, we use 27-connected lattice in 3D, i.e., diagonal propagations are also included for better accuracy. In addition, the medialness measure is computed orthogonal to the direction of propagation instead of computing at nodes. The discrete path (curve) from a point $P_{i j}$ to source $P_{0}$ can then be easily obtained by traversing (backtracking) along the propagation. 

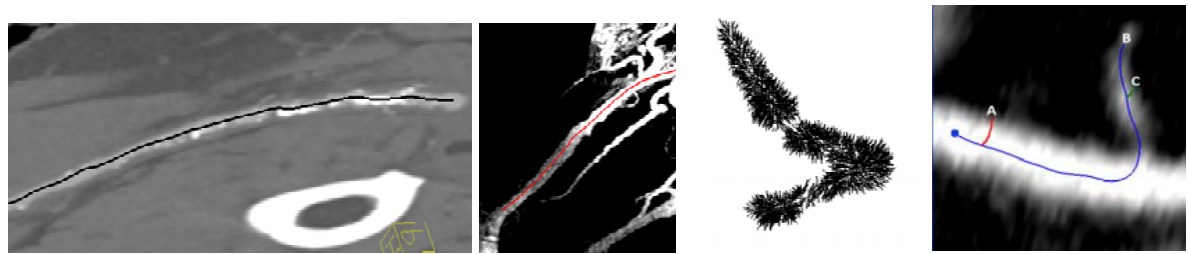

Fig. 3. This figure illustrates the local center axis models of vessels from CTA and MRA. Our algorithm works well even in the presence of nearby vessels, strong calcification and strong contrast change along a vessel.

Fig. 4. (left) The discrete front and centerlines from these front points. (right) The branch removal process. Observe that front point $B$ is kept while front points $A$ are $C$ are removed.

Our major contribution of this minimum-cost path finding algorithm is the use of medialness measure as the cost of a graph edge and its orthogonal computation to the graph edge during propagations. In fact, this orthogonal computation is the key factor for accuracy and increased computational efficiency since the costs obtained on vessel cross-sections are small, i.e., high medialness response. In other words, fronts propagates much faster in the center of vessels and much slower to towards vessel walls due to this fact. In fact, the proposed centerline extraction algorithm may also be viewed as the minimum-cost cross-sectional plane estimation algorithm since the propagation prefers directions which produce better cross-sections. Figure 3 illustrates some centerlines obtained between two seeds from this algorithm. This algorithm works well even in the presence of nearby vessels, strong calcification and strong contrast change along a vessel and it is computationally efficient. For example, a centerline segment of a coronary artery can be obtained from this algorithm in 3 seconds via two seed placements.

\section{Vessel Centerline Tree Extraction}

In this section, we extend the local centerline detection algorithm to recover the full vessel tree from a single point, a source which may be initialized by an user or another process. Recall that the above algorithm terminates when the front propagation reaches to a sink, an end point. When there is no sink point defined for an explicit stopping, the propagation should continue until it reaches to all the branches. The stopping criteria that we choose in our algorithm is based on the medialness measure along a discrete front. Specifically, propagation is forced to stop when the minimum medialness measure along a discrete front at any time drops below a threshold. In our experiments, we found this stopping criteria to be very reliable in clinical applications since our medialness measure is designed to be very low outside vessels. However, the total occlusion cases, where piece of a vessel is totally closed, require starting the propagation on the other side of an occlusion, manually or automatically. We first illustrate how to determine the correct vessel centerline tree from the converged propagation.

Suppose that the propagation has converged at time $t_{f}$ with a set of graph nodes, $F=\left(P_{1}, \ldots, P_{K}\right)$, representing a discrete front $F$, Figure 4. A 
minimum-cost path between each point $P_{i}$ of a discrete front, $F$ and the source $P_{0}$ can be computed from the minimum accumulative cost map, $\phi$, resulting in $K$ different paths. It is obvious that most of these paths are redundant, i.e., a single vessel branch should represented by a single centerline or a single front point. In addition, the existence of a vessel branch can be determined by its length, $L_{B}$ and its approximate radius, $R_{B}$ along its centerline, $C$, i.e., $L_{B}>>R_{B} 11$. Let us illustrate the basic idea of selecting one centerline for each vessel branch via an example, in Figure $4 \mathrm{~b}$ which depicts three points $A, B, C$ on a vessel boundary and their corresponding minimum-cost paths. It is clear that the point $B$ with its path $C_{B}$ represents a branch while the front point $A$ does not since the length of its path is similar to its radius. The front point $C$ may be considered as representing a vessel branch since the length of its minimal path to the source $P_{0}$ is significant relative to its average radius. However, the path $C_{B}$ represents the vessel branch better than the path $C_{C}$ starting from $C$. These observations suggest that a front point with the longest path represents a vessel branch better when there are several front points on the same vessel boundary, which is the case after stopping the propagation. This can be implemented very efficiently with the following algorithm:

1. compute the minimum-cost path $C_{i}$ and the length $L_{i}$ for each point $P_{i}$ in the discrete front set $F$.

2. compute the average radius, $R_{C}$ along the each path $C_{i}$ from the scale information contained in the medialness filters.

3. order the paths based on their length and store them in a queue, $Q_{C}$, i.e., maximum is on top.

4. continue until the queue, $Q_{C}$ is empty

(a) select the path $C$ from the top of the queue and remove it from the queue.

(b) recompute the path by backtracking until the source, $P_{0}$ or the previously computed path on the minimum-accumulative cost map is encountered

(c) mark the path in the minimum-accumulative cost map during the tracking process

(d) recompute the length of the new path, $L_{C}$

(e) set the saliency of the path $C$ or its corresponding front point, $P$ as $L_{C} / R_{C}$

5. delete the paths whose saliency is less than a user-defined threshold,

In our experiments, the saliency threshold is set to 2.0, which means that length of a vessel branch should be two times greater than its average radius along its centerline, otherwise it does not appear to be a significant vessel branch. Figure 5 illustrates some examples of vessel centerline tree for coronary arteries and cerebral vessels and others.

\section{Results and Validations}

We have tested our centerline detection algorithm on coronary, carotid, aorta, peripheral, cerebral, and other vessels obtained from CTA, MRA and 3D rotational angiography data. Figure [5] illustrates some of the results which are

\footnotetext{
${ }^{1}$ The length of a centerline, $C$, is given by $L_{C}=\int_{C} d s$ where $s$ is the arc length.
} 

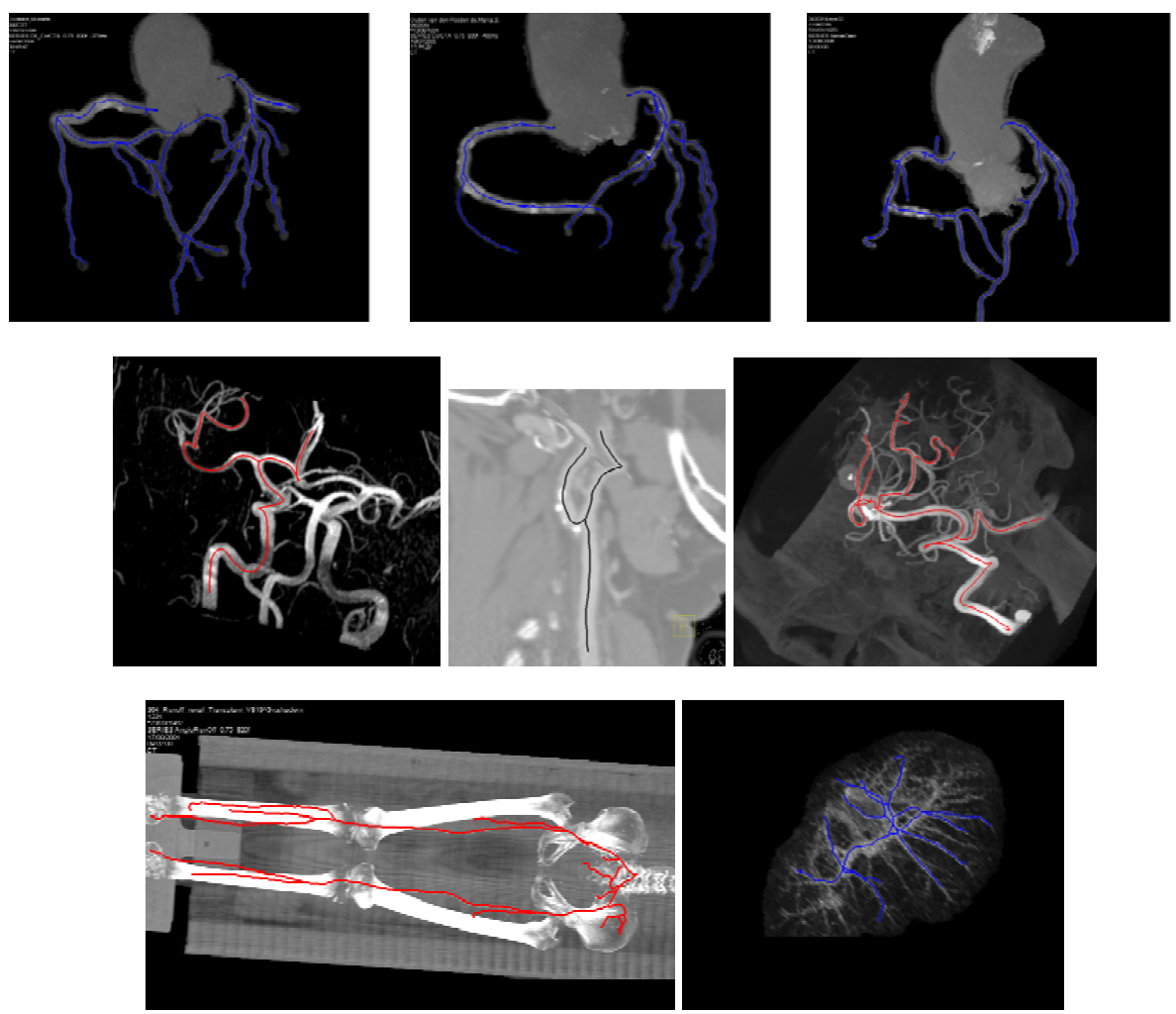

Fig. 5. This figure illustrates the results obtained from our algorithm. Top row: coronary arteries obtained from CTA. Centerlines are drawn in blue and coronary vessel masks are created by using the scales contained in centerline trees. Middle row: cerebral vessels from MRA, CTA and 3D rotational angiography Bottom row: peripheral vessels from CTA and liver vessels after liver segmentation.

obtained by initializing a single seed on a vessel. In our validation studies, coronary arteries from CTA were our main focus where the accuracy of vessel detection (structural validation) was measured and the computed centerline locations were compared with the expert created ground-truth. Specifically, there were 100 coronary artery CTA data in the experiment. The centerline tree extraction algorithm started from the ostia points, namely the beginning of coronary arteries from aorta, which were automatically determined by an aorta detection algorithm. If there were errors in the locations of such ostia points, the user were given tools to correct them. In the structural validations, an expert visualized the detected centerlines on original data and quantified the number of correctly detected arteries. The following detection ratios were obtained: left coronary artery (LCA) \%100, right coronary artery (RCA) \%100, left anterior descending artery (LAD) \%95, left circumflex branch (LCX) \%93, Left Acute Marginal \%94, Obtuse Marginal, \%92, where a failure is marked when a centerline went outside a certain artery or it was not able to reach to the end of a branch. It should be 
Table 1. This table illustrates the error ( $\mathrm{mm}$ ) between computed and expertconstructed vessel centerlines

\begin{tabular}{|l|c|c|c|c|}
\hline Vessel Type - Error (mm) & Average & Standard Deviation & Maximum & No of Data Set \\
\hline \hline Coronary: LCA (CTA) & 0.33 & 0.14 & 0.74 & 8 \\
\hline Coronary: RCA (CTA) & 0.30 & 0.12 & 0.55 & 8 \\
\hline Coronary: LCX (CTA) & 0.28 & 0.15 & 0.59 & 8 \\
\hline Coronary: LAD (CTA) & 0.31 & 0.16 & 0.67 & 8 \\
\hline Carotid (CTA) & 0.45 & 0.21 & 1.08 & 3 \\
\hline Cerebral (MRA) & 0.49 & 0.29 & 1.13 & 3 \\
\hline peripheral (CTA) & 0.64 & 0.42 & 3.59 & 3 \\
\hline
\end{tabular}

noted that it took an average of 21 seconds to construct such trees from CTA data sets on a $3.2 \mathrm{GHz} \mathrm{PC}$.

In addition to these validations, we have also quantified the errors between expert created centerlines and the computed centerlines by measuring the distances between each corresponding centerline. Specifically, we first designed a manual vessel centerline construction tool where an user can easily and correctly create vessel centerlines. Table 1 illustrates the quantitative results on different types of vessels obtained from both CTA and MRA. Interestingly, similar errors were observed between the results obtained from two different experts.

\section{Conclusions}

In this paper, we presented a novel algorithm for the extraction of center axis representations for the blood vessels found in different imaging modalities such as CTA, MRA and 3D X-Ray data. The algorithm is suitable for clinical applications for diagnosis, treatment planning and follow-up studies of vascular structures.

\section{References}

1. Aylward, S., Eberly, E.: Initialization, noise, singularities, and scale in height-ridge traversal for tubular object centerline extraction. TMI 21(2), 61-75 (2002)

2. Aylward, S., Pizer, S., Bullitt, E., Eberly, D.: Intensity ridge and widths for 3d object segmentation and description. In: IEEE Proc. Workshop MMBIA, pp. 131138 (1996)

3. Deschamps, T., Cohen, L.: Fast extraction of minimal paths in 3d images and applications to virtual endoscopy. Medical Image Analysis 5(4), 281-299 (2001)

4. Dijkstra, E.W.: A note on two problems in connections with graphs. Numerische Mathematic 1, 269-271 (1959)

5. Frangi, A.F., Niessen, W.J., Vincken, K.L., Viergever, M.A.: Multiscale vessel enhancement filtering. In: Wells, W.M., Colchester, A.C.F., Delp, S.L. (eds.) MICCAI 1998. LNCS, vol. 1496, pp. 82-89. Springer, Heidelberg (1998)

6. Koller, T.M., Gerig, G., Szekely, G., Dettwiler, D.: Multiscale detection of curvilinear structures in 2-D and 3-D image data. In: ICCV, pp. 864-869 (1995)

7. Krissian, K., Malandain, G., Ayache, N., Vaillant, R., Trousset, Y.: Model based multiscale detection of 3D vessels. In: IEEE Conf. CVPR, pp. 722-727 (1998) 
8. Li, H., Yezzi, A.J.: Vessels as 4-D curves: Global minimal 4-D paths to extract 3-D tubular surfaces and centerlines. IEEE Trans. Med. Imaging 26(9), 1213-1223 (2007)

9. Sethian, J.A.: Level Set Methods. Cambridge University Press, New York (1996)

10. Siddiqi, K., Vasilevskiy, A.: 3D flux maximizing flows. In: International Workshop on Energy Minimizing Methods In Computer Vision (2001)

11. Tyrrell, J.A., di Tomaso, E., Fuja, D., Tong, R., Kozak, K., Brown, E.B., Jain, R., Roysam, B.: Robust 3-D modeling of vasculature imagery using superellipsoids. IEEE Transactions on Medical Imaging (2006)

12. Wink, O., Niessen, W.J., Viergever, M.A.: Multiscale vessel tracking. IEEE Trans. on Medical Imaging 23(1), 130-133 (2004) 